\title{
新型含二硫环戊烯酮结构新烟碱类衍生物的合成与生物活性
}

\author{
田忠贞王先浩李冬梅*
}

(内蒙古科技大学化学与化工学院 内蒙古自治区煤化工与煤炭综合利用重点实验室 内蒙古包头 014010)

\begin{abstract}
摘要 为了探索与发现具有优异活性的内酯类新烟碱杀虫剂, 利用骨架跃迁引入二硫环戊烯酮新药效团, 设计合成了 一系列结构新颖的 1,2-二硫环戊烯酮类新烟碱化合物. 所合成化合物结构均经核磁共振(NMR)和高分辨质谱(HRMS)等 方法确证. 初步生物活性测试表明, 目标化合物对苜宿蚜虫和褐飞虫都具有一定的杀虫活性，其中 4-氯-5-(((6-氯吡啶3-基)甲基)(甲基)氨基)-3H-1,2-二硫醇-3-酮(3a), 4-氯-5-(((6-氯吡啶-3-基)甲基)(乙基)氨基)-3H-1,2-二硫醇-3-酮(3b), 4-氯5-(((6-氯吡啶-3-基)甲基)(丙基)氨基)-3H-1,2-二硫醇-3-酮(3c)和 4-氯-5-(((6-氯吡啶-3-基)甲基)(2,2-二氟乙基)氨 基)-3H-1,2-二硫醇-3-酮(3g)在 $100 \mu \mathrm{g} / \mathrm{mL}$ 浓度下对苗宿蚜虫的致死率均超过 $90 \%, \mathbf{3 g}$ 在 $4 \mu \mathrm{g} / \mathrm{mL}$ 对苜宿蚜具有 $40 \%$ 的致 死率. 此外 $3 \mathrm{~g}$ 在 $100 \mu \mathrm{g} / \mathrm{mL}$ 浓度下对稻飞虫的致死率超过 $70 \%$, 可做深入的结构优化与杀虫活性探究.
\end{abstract}

关键词 新烟碱杀虫剂; 二硫环戊烯酮; 合成; 杀虫活性

\section{Synthesis, Bioactivities of Novel Neonicotinoid Derivatives Containing 1,2-Dithiocyclopentenone Moiety}

\author{
Tian, Zhongzhen Wang, Xianhao Li, Dongmei* \\ (Inner Mongolia Key Laboratory of Coal Chemical Engineering \& Comprehensive Utilization, School of Chemistry and \\ Chemical Engineering, Inner Mongolia University of Science \& Technology, Baotou, Mongolia 014010)
}

\begin{abstract}
In search of novel neonicotinoid insecticides with potent bioactivities, a series of 1,2-dithiocyclopentenone derivatives were designed and synthesized by introducing dithiocyclopentenone into neonicotinoids skeleton. The structures of all compounds were confirmed by nuclear magnetic resonance (NMR) and high resolution mass spectrometry (HRMS). Biological activities of all the title compounds were evaluated systematically. Preliminary bioassays indicated that all synthesized compounds exhibited moderate insecticidal activities. 4-Chloro-5-(((6-chloropyridin-3-yl)methyl)(methyl)amino)-3H-1,2-dithiol-3-one (3a), 4-chloro-5-(((6-chloropyridin-3-yl)methyl)(ethyl)amino)-3H-1,2-dithiol-3-one (3b), 4-chloro-5-(((6-chloropyridin-3-yl)methyl)(propyl)amino)-3H-1,2-dithiol-3-one (3c) and 4-chloro-5-(((6-chloropyridin-3-yl)methyl)(2,2-difluoroethyl)amino)-3H-1,2-dithiol-3-one (3g) exhibited $>90 \%$ mortality rate against Aphis craccivora at $100 \mu \mathrm{g} / \mathrm{mL}$. Compound $\mathbf{3 g}$ exhibited $40 \%$ insecticidal activity to aphids at $4 \mu \mathrm{g} / \mathrm{mL}$ and $>70 \%$ mortality rate against Nilaparvata Lugens at $100 \mu \mathrm{g} / \mathrm{mL}$, and could be utilized in insecticide research with further optimization.
\end{abstract}

Keywords neonicotinoid insecticide; dithiocyclopentenone; synthesis; insecticidal activity

新烟碱类化合物是一类作用于昆虫烟碱乙酰胆碱 受体(nAChR)的杀虫剂 ${ }^{[1]}$, 因其高效、广谱、低毒等优势 成为全球杀虫剂市场的重要组成部分 ${ }^{[2-3]}$. 但随着传统 新烟碱类杀虫剂在世界范围内的长期广泛使用，一系列 问题和弊端也随之浮现. 研究者们发现传统新烟碱类杀 虫剂如吡虫啉(Imidacloprid, 图 1)和噻虫嗪(Thiamethoxam, 图 1)等对蜜蜂毒性为高毒或剧毒, 且具有亚致
死效应; 其有效成分及代谢物在花粉和花蜜中残留，导 致蜜蜂种群减少, 繁殖能力降低 ${ }^{[4]}$. 鉴于此, 多个国家 和组织对部分新烟碱类杀虫剂的环境风险进行了再评 价或限制使用, 甚至禁用. 而新烟碱类杀虫剂就目前甚 至较长时间内均不存在完全的替代产品, 开发针对蜜蜂 等传粉昆虫安全的结构新颖的新烟碱杀虫剂尤为紧迫. 2018 年在中国取得登记的氟吡呋喃酮(Flupyradifurone,

\footnotetext{
* Corresponding author. E-mail: 2019951@imust.edu.cn

Received June 26, 2020; revised July 21, 2020; published online August 11, 2020.

Project supported by the Natural Science Foundation of Shandong Province (No. ZR2018MB013), the National Natural Science Foundation of China (No. 21501066) and the Opening Project of Shanghai Key Laboratory of Chemical Biology.

山东省自然科学基金(No. ZR2018MB013)、国家自然科学基金(No. 21501066)和上海市化学生物学(芳香杂环)重点实验室资助项目.
} 


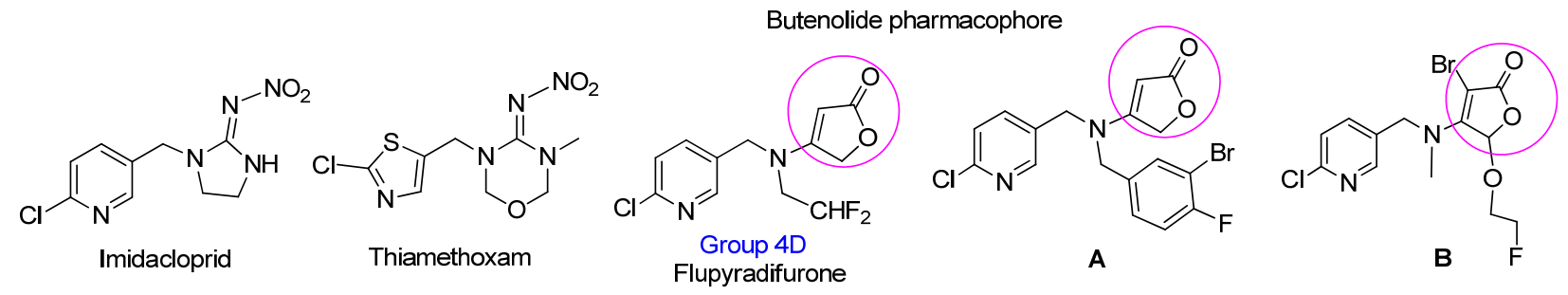

图 1 化合物 A、B、吡虫啉、噻虫嗪和氟吡呋喃酮和的化学结构式

Figure 1 Chemical structures of compounds A, B, imidacloprid, thiamethoxam and flupyradifurone

图 1), 归类于 Group 4D 激动剂, 具有全新的药效团结构 “丁烯酸内酯”和独特的杀虫作用机制，且与其它烟碱型 乙酰胆碱激动剂不存在交互抗性 ${ }^{[5-6]}$. 其新颖的药效团 结构被认为起到关键性作用, 灵感源于百部叶碱分子 (Stemofoline)结构 ${ }^{[7-8]}$. 同时氟吡呋喃酮对蜜蜂的急性接 触 $\mathrm{LD}_{50}>200 \mu \mathrm{g} / \mathrm{bee}$, 是目前世界上公认对蜜蜂安全的 新烟碱杀虫剂 ${ }^{[9-10]}$, 即使在作物开花期, 也可喷雾用药. 因此，该化合物一经问世便受到世界农药研究者的广泛 关注, 一系列围绕此结构的研究和衍生探索也在持续进 行中 ${ }^{[11]}$.

2018 年报道的苄基取代的丁烯酸内酯类新烟碱化 合物 $\mathbf{A}$, 对蚕豆蚜 $\mathrm{LC}_{50}$ 值为 $1.72 \mu \mathrm{g} / \mathrm{mL}^{[12]} .2019$ 年本课 题组 ${ }^{[13]}$ 报道的 $\gamma$-烷氧基- $\alpha$-溴丁烯酸内酯新烟碱化合物 B, 在 $10 \mathrm{mg} / \mathrm{L}$ 条件下对稻飞闽和蚜虫的死亡率均超过 90\%. Group 4D 亚组业已成为新烟碱杀虫剂新的研发热 点, 但丁烯酸内酯药效团可修饰性限制其进一步发展, 寻找新的内酯类药效团则尤为重要.

骨架跃迁(Scaffold Hopping)用于改变药效团结构以
寻找新药方面具有重要的应用, 往往可以获得生理作用 相似或相拮抗的化合物, 并且骨架跃迁可以有效避开现 有专利, 有利于原创农药的研发. 4,5-二氯-1,2-二硫环戊 烯酮是高效杀微生物剂 ${ }^{[14-15]}$, 被广泛应用于供水系统、 杀菌剂、杀虫剂、化妆品、家居用品等领域(图 2). 而该 类化合物具有独特二硫环戊烯酮骨架, 理论上与丁烯酸 内酯药效团是生物电子等排体，但具有不同的合成路线 和起始原料. 该化合物价格低廉，对农药的生产成本控 制具有明显优势. 在新烟碱杀虫剂发展二十几年里, 也 未见二硫环戊烯酮骨架应用于新烟碱杀虫剂的创制. 因 此，本工作利用二硫环戊烯酮代替氟吡呋喃酮中的丁烯 酸内酯药效团, 通过中间体和二氯-1,2-二硫环戊烯酮的 迈克尔加成一消除反应得到一系列未经文献报道的化合 物, 并对其杀虫活性进行了初步研究.

\section{1 结果与讨论}

\section{1 目标化合物的合成}

如 Scheme 1 所示, 以 2-氯-5-氯甲基吡啶和脂肪胺

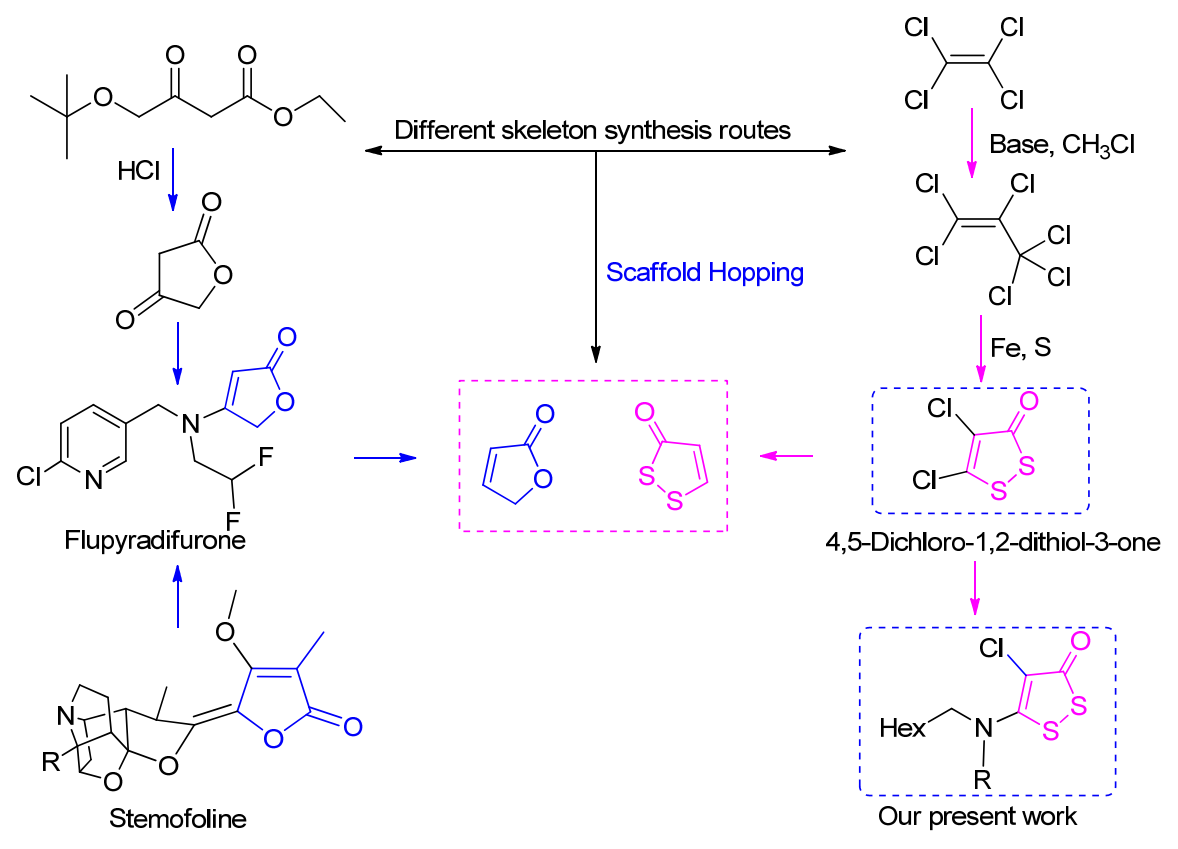

图 2 目标化合物的设计策略

Figure 2 Design strategy of target compounds 

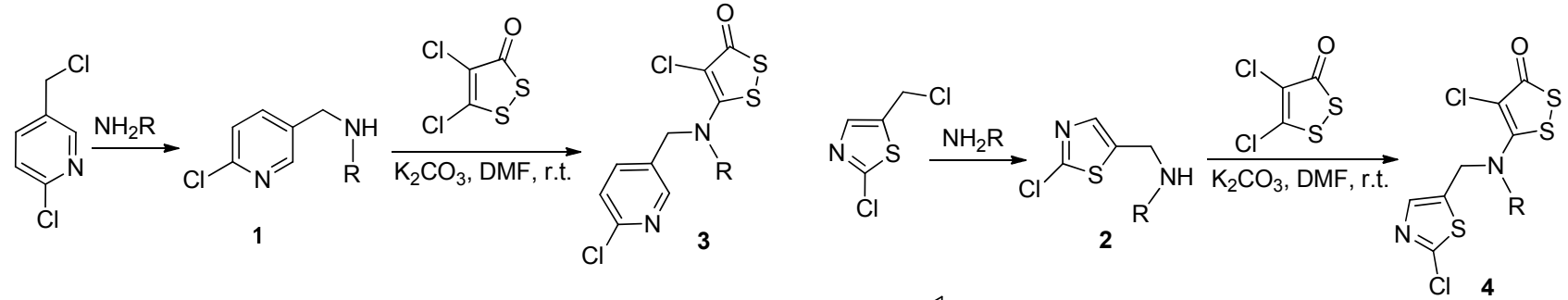

3a: $\mathrm{R}=\mathrm{CH}_{3} ; \mathbf{3 b}: \mathrm{R}=\mathrm{CH}_{2} \mathrm{CH}_{3} ; \mathbf{3 c}: \mathrm{R}=\mathrm{CH}_{2} \mathrm{CH}_{2} \mathrm{CH}_{3} ; \mathbf{3 d}: \mathrm{R}=\mathrm{CH}\left(\mathrm{CH}_{3}\right)_{2} ; \mathbf{3 e}: \mathrm{R}=-\mathrm{q} ; 3 \mathbf{f}: \mathrm{R}=\mathrm{CH}_{2} \mathrm{CH}_{2} \mathrm{CH}_{2} \mathrm{CH}_{3} ; \mathbf{3 g}: \mathrm{R}=\mathrm{CH}_{2} \mathrm{CHF}_{2}$;
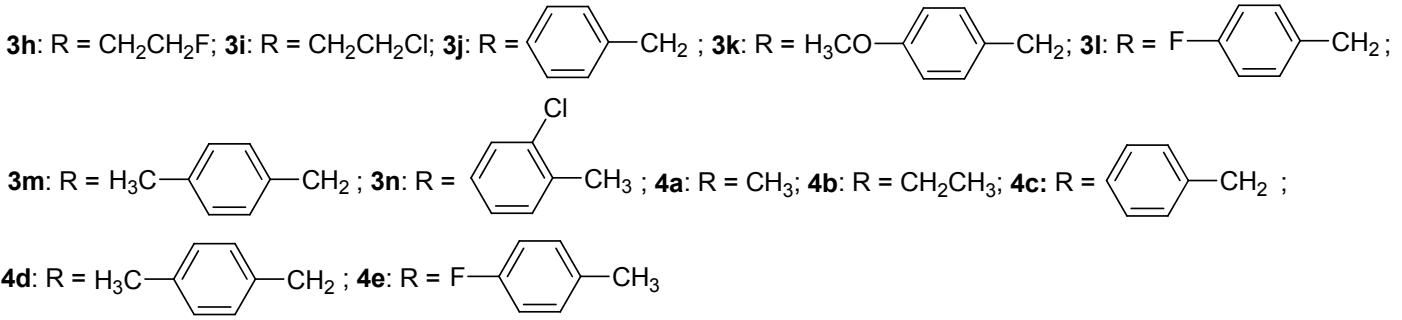

图式 1 目标化合物 3 和 4 的合成路线

Scheme 1 Synthetic routes of target compounds 3 and 4

或苄胺为原料, 常温下经取代反应得到中间体 1 . 中间 体 1 再与二氯-1,2-二硫环戊烯酮在 $N, N$-二甲基甲酰胺 (DMF)中经迈克尔加成-消除反应得到目标化合物 3 . 以 2-氯-5-氯甲基噻唑和脂肪胺或芐胺为原料, 经取代反应 得到中间体 2, 再与二氯-1,2-二硫环戊烯酮在 DMF 中反 应，得到目标化合物 4.

\section{2 化合物波谱分析}

以化合物 3a 为例进行谱图分析, ${ }^{1} \mathrm{H}$ NMR 谱图中 $\delta$ $8.36,7.67,7.39$ 处的二重峰及双二重峰为吡啶环上氢吸 收峰; $\delta 4.86$ 处的单峰为亚甲基上氢的吸收峰; $\delta 3.16$ 处 的单峰为甲基上氢的吸收峰. ${ }^{13} \mathrm{C}$ NMR 谱图中, $\delta 183.3$ 处的峰为羰基碳的信号峰; $\delta 167.5,100.0$ 处的峰为环伐 烯酮内双键两端碳原子的信号峰; $\delta 151.6,148.9,138.0$, 130.2124 .7 处的峰为吡啶环上碳原子的信号峰; $\delta 54.8$ 处的峰为亚甲基碳原子的信号峰; $\delta 39.8$ 处的峰为甲基 碳原子的信号峰. HRMS 谱图中, 该化合物分子离子峰 计算值为 $[\mathrm{M}+\mathrm{H}]^{+}$306.9533, 测定值 $[\mathrm{M}+\mathrm{H}]^{+}$306.9531, 绝对误差在 0.0030 以内.

\section{3 化合物的生物活性及构效关系分析}

目标化合物对苜宿蚜虫和稻飞風的杀虫活性列于 表 1 . 由表 1 数据可见, 所有化合物在 $100 \mu \mathrm{g} / \mathrm{mL}$ 浓度下 均对苜葆蚜虫有一定的杀虫活性, 3a 3n 以及 $4 a, 4 b$ 对 苜葆蚜虫的致死率均在 $40 \% \sim 100 \%$ 之间. 其中化合物 3a, 3b, 3c 以及 $3 \mathrm{~g}$ 的杀虫效果较为突出, 致死率分别达 到 $100 \%, 100 \%, 93.4 \%$ 和 $91.8 \%$. 在 $100 \mathrm{mg} / \mathrm{L}$ 浓度下大 部分化合物对稻飞風有一定的杀虫活性，但整体活性较 低. 其中化合物 $3 \mathrm{a}$ 和 $3 \mathrm{~g}$ 对稻飞虫的致死率分别为 $66.7 \%$ 和 $71.8 \%$, 杀虫活性低于对照药物 Flupyradifu-
表 1 目标化合物的杀虫活性(死亡率\%) $)^{a}$

Table 1 Insecticidal activities of the target compounds (mortality $\%$ )

\begin{tabular}{|c|c|c|c|c|}
\hline \multirow{2}{*}{ Compd. } & \multicolumn{3}{|c|}{ Aphis medicaginis } & \multirow{2}{*}{$\frac{\text { Nilaparvata Lugens }}{100 \mu \mathrm{g} / \mathrm{mL}}$} \\
\hline & $100 \mu \mathrm{g} / \mathrm{mL}$ & $20 \mu \mathrm{g} / \mathrm{mL}$ & $4 \mu \mathrm{g} / \mathrm{mL}$ & \\
\hline $3 a$ & 100.0 & 41.9 & 22.6 & 66.7 \\
\hline $\mathbf{3 b}$ & 100.0 & 46.0 & 24.0 & 32.6 \\
\hline $3 c$ & 94.3 & 37.3 & 28.4 & 27.5 \\
\hline 3d & 45.7 & - & - & 24.6 \\
\hline $3 e$ & 61.1 & - & - & 28.7 \\
\hline $3 f$ & 43.4 & - & - & 19.6 \\
\hline $3 g$ & 91.8 & 50.4 & 40.8 & 71.8 \\
\hline $3 \mathbf{h}$ & 45.9 & - & - & 38.8 \\
\hline $3 \mathbf{i}$ & 63.4 & - & - & 26.5 \\
\hline $3 \mathbf{j}$ & 60.9 & - & - & 21.1 \\
\hline $3 \mathbf{k}$ & 59.1 & - & - & 18.7 \\
\hline 31 & 56.8 & - & - & 19.3 \\
\hline $3 \mathrm{~m}$ & 84.5 & - & - & 50.3 \\
\hline $3 n$ & 68.4 & - & - & 30.8 \\
\hline $4 a$ & 64.6 & - & - & 48.2 \\
\hline $4 b$ & 55.2 & - & - & 44.7 \\
\hline $4 c$ & 15.3 & - & - & 14.0 \\
\hline $4 d$ & 22.5 & - & - & 20.9 \\
\hline $4 e$ & 22.5 & - & - & 56.6 \\
\hline $\begin{array}{l}\text { Flupyra- } \\
\text { difurone }\end{array}$ & 100.0 & 100.0 & 95.0 & 90.0 \\
\hline
\end{tabular}

rone. 对活性较高的化合物 $3 \mathrm{a}, 3 \mathrm{~b}, 3 \mathrm{c}$ 和 $3 \mathrm{~g}$ 进行低浓度 复篎，当浓度降至 $20 \mu \mathrm{g} / \mathrm{mL}$ 时, 3a, 3b 和 3c 对苜宿蚜虫 的致死率在 $40 \%$ 左右, $3 \mathrm{~g}$ 的致死率为 $50 \%$; 当浓度降至 
$4 \mu \mathrm{g} / \mathrm{mL}$ 时, 3a, $3 \mathbf{b}$ 和 $\mathbf{3 c}$ 的致死率均只有 $20 \%$ 左右, $\mathbf{3 g}$ 的致死率为 $40 \%$.

初步构效关系分析可知，当取代基 $\mathrm{R}$ 为甲基、乙基、 正丙基以及 2,2-二氟乙基时, 对应化合物的杀虫活性远 高于其他化合物; 当 $\mathrm{R}$ 基为 2 -氟乙基和 2 -氯乙基时，对 应杀虫活性低于 2,2-二氟乙基，且 2-氯乙基取代的化合 物杀虫活性低于 2 -氟乙基. 取代基 $\mathrm{R}$ 为芳香基时, 对应 化合物的杀虫活性略优于异、环丙基以及丁基; 而苯环 上有无取代基以及取代基的种类对杀虫活性并无明显 影响. 当取代基 $\mathrm{R}$ 一定时, 杂环为吡啶的化合物杀虫活 性高于杂环为噻唑的化合物. 另外, 对比低浓度下化合 物 3a, 3b, 3c 和 3g 的活性数据可知, 化合物取代基中氟 原子的引入对杀虫活性有积极的影响. 由化合物 $\mathbf{3 g}, \mathbf{3 m}$ 和 3n 的活性数据对比可知, 当引入氟原子个数减少时, 对应化合物的杀虫活性会降低, 若将氟原子替换为其他 卤族原子如氯原子时, 杀虫活性会进一步降低.

整体来看, 所有化合物对苜葆蚜虫的杀灭活性高于 褐飞風, 但大部分化合物对两种供试害虫的杀虫活性较 低. 其中化合物 $3 a, 3 b, 3 c$ 和 $3 g$ 对苜宿蚜虫的杀虫活性 较突出，由简单构效关系分析可知: $\mathrm{R}$ 基为甲基、乙基、 正丙基和 2,2-二氟乙基时化合物对苜葆蚜虫有较好的杀 灭活性.

\section{2 结论}

以 2-氯-5-氯甲基吡啶、2-氯-5-氯甲基噻唑和二氯1,2-二硫环戊烯酮等为原料, 经取代反应、迈克尔加成消除反应合成了一系列结构新颖的 1,2-二硫环戊烯酮衍 生物. 初步生物活性测试结果表明, 所有化合物均对苜 葆蚜虫和稻飞風有一定的杀虫活性, 但整体活性较低. 化合物 3a, 3b, 3c 和 $3 \mathrm{~g}$ 在 $100 \mu \mathrm{g} / \mathrm{mL}$ 浓度下对苜宿蚜虫 的致死率大于 $90 \%$, 其中 $\mathbf{3 a}$ 和 $\mathbf{3 b}$ 在 $100 \mu \mathrm{g} / \mathrm{mL}$ 的浓度 下对苜葆蚜虫的致死率达到 $100 \%$. 因此利用 1,2-二硫 环戊烯酮结构作为新烟碱类杀虫剂的药效基团具有深 入研究的价值.

\section{3 实验部分}

\section{1 仪器与试剂}

RY-1 型熔点仪(天津分析仪器厂); Avance III 400 $\mathrm{MHz}$ 核磁共振仪 $\left(\mathrm{CDCl}_{3}\right.$ 为溶剂, $\mathrm{TMS}$ 为内标, 德国 Bruker 公司); Varian QFT-ESI 质谱仪(美国 Varian 公司). 所有试剂均为国产分析纯.

\section{2 实验方法}

\subsection{1中间体化合物 $\mathbf{1}$ 和 $\mathbf{2}$ 的合成}

参照文献[16]合成中间体化合物 $\mathbf{1}$ 和 $\mathbf{2}$, 无需处理直 接用于下步反应.

\subsection{2 目标化合物 $3 \mathbf{a} \sim 3 \mathbf{n}$ 的合成}

$50 \mathrm{~mL}$ 圆底烧瓶中依次加入中间体 $\mathbf{1}(4 \mathrm{mmol})$ 、二 氯-1,2-二硫环戊烯酮 $(2.7 \mathrm{mmol})$ 与 $3 \mathrm{~mL}$ DMF, 搅拌使其 完全溶解. 混合液加入无水碳酸钾 $0.35 \mathrm{~g}$, 室温搅拌, 薄层色谱(TLC)跟踪反应. 反应结束后向反应液中加入 $50 \mathrm{~mL}$ 冰水, 抽滤, 将滤饼溶于 $50 \mathrm{~mL}$ 二氯甲烷, 经 1 $\mathrm{mol} / \mathrm{L}$ 盐酸溶液、饱和碳酸氢钠溶液和清水洗涤, 干燥 后减压浓缩, 粗产品经柱层析分离 $[V$ (二氯甲烷)： $V($ 乙 酸乙酯 $)=10 ：$ ] 纯化得到目标化合物 $\mathbf{3 a} \sim \mathbf{3 n}$.

4-氯-5-(((6-氯吡啶-3-基)甲基)(甲基)氨基)-3H-1,2二硫醇-3-酮(3a)：淡黄色固体，产率 60\%. m.p. 95 $96{ }^{\circ} \mathrm{C} ;{ }^{1} \mathrm{H}$ NMR $\left(400 \mathrm{MHz}, \mathrm{CDCl}_{3}\right) \delta: 8.36(\mathrm{~d}, J=2.5 \mathrm{~Hz}$, $1 \mathrm{H}), 7.67$ (dd, $J=8.2,2.6 \mathrm{~Hz}, 1 \mathrm{H}), 7.39(\mathrm{dd}, J=8.2,0.7$ $\mathrm{Hz}, 1 \mathrm{H}), 4.86$ (s, 2H), 3.16 (s, 3H); ${ }^{13} \mathrm{C}$ NMR (101 MHz, $\left.\mathrm{CDCl}_{3}\right) \delta: 183.3,167.5,151.6,148.9,138.0,130.2,124.7$, 100.0, 54.8, 39.8; HRMS (ESI) calcd for $\mathrm{C}_{10} \mathrm{H}_{9} \mathrm{Cl}_{2} \mathrm{~N}_{2} \mathrm{OS}_{2}$ $[\mathrm{M}+\mathrm{H}]^{+}$306.9533, found 306.9531 .

4-氯-5-(((6-氯吡啶-3-基)甲基)(乙基)氨基)-3H-1,2二硫醇-3-酮(3b): 淡黄色固体, 产率 54\%. m.p. 93 $94{ }^{\circ} \mathrm{C} ;{ }^{1} \mathrm{H}$ NMR $\left(400 \mathrm{MHz}, \mathrm{CDCl}_{3}\right) \delta: 8.37(\mathrm{~d}, J=2.6 \mathrm{~Hz}$, $1 \mathrm{H}), 7.66(\mathrm{dd}, J=8.2,2.6 \mathrm{~Hz}, 1 \mathrm{H}), 7.40(\mathrm{~d}, J=8.3 \mathrm{~Hz}$, $1 \mathrm{H}), 4.87$ (s, 2H), 3.63 (q, $J=7.1 \mathrm{~Hz}, 2 \mathrm{H}), 1.34$ (t, $J=7.1$ $\mathrm{Hz}, 3 \mathrm{H}) ;{ }^{13} \mathrm{C}$ NMR $\left(101 \mathrm{MHz}, \mathrm{CDCl}_{3}\right) \delta: 183.4,166.3$, 151.5, 148.7, 137.8, 130.6, 124.7, 100.4, 52.2, 47.8, 13.4; HRMS (ESI) calcd for $\mathrm{C}_{11} \mathrm{H}_{11} \mathrm{Cl}_{2} \mathrm{~N}_{2} \mathrm{OS}_{2}[\mathrm{M}+\mathrm{H}]^{+}$ 320.9690 , found 320.9686 .

4-氯-5-(((6-氯吡啶-3-基)甲基)(丙基)氨基)-3 H-1,2二硫醇-3-酮(3c)：暗黄色固体，产率 48\%. m.p. 89 $91{ }^{\circ} \mathrm{C} ;{ }^{1} \mathrm{H}$ NMR $\left(400 \mathrm{MHz}, \mathrm{CDCl}_{3}\right) \delta: 8.35(\mathrm{~d}, J=2.5 \mathrm{~Hz}$, 1H), $7.64(\mathrm{dd}, J=8.2,2.6 \mathrm{~Hz}, 1 \mathrm{H}), 7.39(\mathrm{~d}, J=8.2 \mathrm{~Hz}$, $1 \mathrm{H}), 4.88(\mathrm{~s}, 2 \mathrm{H}), 3.52 \sim 3.46(\mathrm{~m}, 2 \mathrm{H}), 1.75$ (q, $J=7.6 \mathrm{~Hz}$, $1 \mathrm{H}), 0.95$ (t, $J=7.4 \mathrm{~Hz}, 2 \mathrm{H}) ;{ }^{13} \mathrm{C}$ NMR $\left(101 \mathrm{MHz}, \mathrm{CDCl}_{3}\right)$ $\delta: 183.5,166.5,151.5,148.7,137.8,130.6,124.7,100.5$, 54.8, 53.0, 21.6, 11.0; HRMS(ESI): calcd for $\mathrm{C}_{12} \mathrm{H}_{13} \mathrm{Cl}_{2}-$ $\mathrm{N}_{2} \mathrm{OS}_{2}[\mathrm{M}+\mathrm{H}]^{+}$334.9846, found 334.9838 .

4-氯-5-(((6-氯吡啶-3-基)甲基)(异丙基)氨基)- $3 \mathrm{H}$ 1,2-二硫醇-3-酮 (3d): 浅棕色固体，产率 55\%. m.p. 106 108 ${ }^{\circ} \mathrm{C} ;{ }^{1} \mathrm{H}$ NMR (400 MHz, $\left.\mathrm{CDCl}_{3}\right) \delta: 8.35$ (d, $J=$ 2.5, $0.9 \mathrm{~Hz}, 1 \mathrm{H}), 7.59(\mathrm{dd}, J=8.3,2.6 \mathrm{~Hz}, 1 \mathrm{H}), 7.34(\mathrm{~d}$, $J=8.3 \mathrm{~Hz}, 1 \mathrm{H}$ ), 4.65 (s, 2H), 4.47 (q, $J=6.7 \mathrm{~Hz}, 1 \mathrm{H}), 1.39$ (s, 3H), 1.37 (s, 3H); ${ }^{13} \mathrm{C}$ NMR (101 MHz, $\left.\mathrm{CDCl}_{3}\right) \delta$ : 183.9, 167.7, 151.0, 148.5, 137.3, 131.9, 124.5, 104.4, 55.9, 45.7, 20.7; HRMS (ESI) calcd for $\mathrm{C}_{12} \mathrm{H}_{13} \mathrm{Cl}_{2} \mathrm{~N}_{2} \mathrm{OS}_{2}$ $[\mathrm{M}+\mathrm{H}]^{+}$334.9846, found 334.9832 .

4-氯-5-(((6-氯吡啶-3-基)甲基)(环丙基)氨基)-3 $\mathrm{H}$ - 
1,2-二硫醇-3-酮 (3e): 棕黄色固体，产率 62\%. m.p. $142 \sim 143{ }^{\circ} \mathrm{C} ;{ }^{1} \mathrm{H}$ NMR $\left(400 \mathrm{MHz}, \mathrm{CDCl}_{3}\right) \delta: 8.36(\mathrm{~d}$, $J=2.5 \mathrm{~Hz}, 1 \mathrm{H}), 7.59$ (dd, $J=8.2,2.6 \mathrm{~Hz}, 1 \mathrm{H}), 7.35(\mathrm{~d}, J=$ $8.1 \mathrm{~Hz}, 1 \mathrm{H}), 4.99(\mathrm{~s}, 2 \mathrm{H}), 2.69(\mathrm{~s}, 1 \mathrm{H}), 1.05 \sim 0.85(\mathrm{~m}$, $4 \mathrm{H}) ;{ }^{13} \mathrm{C}$ NMR $\left(101 \mathrm{MHz}, \mathrm{CDCl}_{3}\right) \delta: 183.8,168.8,151.5$, 149.2, 138.4, 130.6, 124.6, 101.2, 53.2, 33.7, 10.7; HRMS (ESI) calcd for $\mathrm{C}_{12} \mathrm{H}_{11} \mathrm{Cl}_{2} \mathrm{~N}_{2} \mathrm{OS}_{2}[\mathrm{M}+\mathrm{H}]^{+}$332.9690, found 332.9675 .

5-(丁基((6-氯吡啶-3-基)甲基)氨基)-4-氯-3H-1,2-二 硫醇-3-酩(3f): 棕黄色固体, 产率 58\%. m.p. $64 \sim 65{ }^{\circ} \mathrm{C}$; ${ }^{1} \mathrm{H}$ NMR $\left(400 \mathrm{MHz}, \mathrm{CDCl}_{3}\right) \delta: 8.35(\mathrm{~d}, J=2.5 \mathrm{~Hz}, 1 \mathrm{H})$, 7.64 (dd, $J=8.2,2.5 \mathrm{~Hz}, 1 \mathrm{H}), 7.39$ (d, $J=8.3 \mathrm{~Hz}, 1 \mathrm{H})$, $4.88(\mathrm{~s}, 2 \mathrm{H}), 3.62 \sim 3.43(\mathrm{~m}, 2 \mathrm{H}), 1.70(\mathrm{tt}, J=9.2,7.3 \mathrm{~Hz}$, $2 \mathrm{H}), 1.31 \sim 1.38(\mathrm{~m}, J=7.4 \mathrm{~Hz}, 2 \mathrm{H}), 0.95(\mathrm{t}, J=7.4 \mathrm{~Hz}$, $3 \mathrm{H}) ;{ }^{13} \mathrm{C}$ NMR $\left(101 \mathrm{MHz}, \mathrm{CDCl}_{3}\right) \delta: 183.4,166.5,151.5$, 148.7, 137.8, 130.6, 124.7, 100.5, 52.9, 52.9, 30.2, 19.8, 13.7; HRMS (ESI) calcd for $\mathrm{C}_{13} \mathrm{H}_{15} \mathrm{Cl}_{2} \mathrm{~N}_{2} \mathrm{OS}_{2}[\mathrm{M}+\mathrm{H}]^{+}$ 349.0003, found 348.9987.

4 -氯-5-(((6-氯吡啶-3-基)甲基)(2,2-二氟乙基)氨 基)-3 $H$-1,2-二硫醇-3-酮(3g): 棕黄色油状液体, 产率 49\%. ${ }^{1} \mathrm{H}$ NMR (400 MHz, $\left.\mathrm{CDCl}_{3}\right) \delta: 8.38(\mathrm{~d}, J=2.4 \mathrm{~Hz}$, $1 \mathrm{H}), 7.65(\mathrm{dd}, J=8.2,2.3 \mathrm{~Hz}, 1 \mathrm{H}), 7.41(\mathrm{~d}, J=8.2 \mathrm{~Hz}$, $1 \mathrm{H}), 6.10$ (tt, $J=55.0,3.8 \mathrm{~Hz}, 1 \mathrm{H}), 4.86(\mathrm{~s}, 2 \mathrm{H}), 3.96(\mathrm{td}$, $J=13.8,3.8 \mathrm{~Hz}, 2 \mathrm{H}) ;{ }^{13} \mathrm{C}$ NMR $\left(101 \mathrm{MHz}, \mathrm{CDCl}_{3}\right) \delta$ : 183.6, 166.6, 152.0, 149.1, 138.0, 129.5, 124.9, $114.4(\mathrm{t}$, $J=245.0 \mathrm{~Hz}), 104.8,54.4,53.5$ (t, $J=26.2 \mathrm{~Hz})$; HRMS (ESI) calcd for $\mathrm{C}_{11} \mathrm{H}_{9} \mathrm{Cl}_{2} \mathrm{~F}_{2} \mathrm{~N}_{2} \mathrm{OS}_{2}[\mathrm{M}+\mathrm{H}]^{+}$356.9501, found 356.9503 .

4-氯-5-(((6-氯吡啶-3-基)甲基)(4-甲氧基苄基)氨 基)-3H-1,2-二硫醇-3-酮(3h): 淡黄色固体，产率 55\%. m.p. 77 79 ${ }^{\circ} \mathrm{C} ;{ }^{1} \mathrm{H}$ NMR $\left(400 \mathrm{MHz}, \mathrm{CDCl}_{3}\right) \delta: 8.24(\mathrm{~d}$, $J=2.5 \mathrm{~Hz}, 1 \mathrm{H}), 7.59$ (dd, $J=8.2,2.5 \mathrm{~Hz}, 1 \mathrm{H}), 7.36$ (d, $J=$ $8.2 \mathrm{~Hz}, 1 \mathrm{H}), 7.26 \sim 7.12(\mathrm{~m}, 2 \mathrm{H}), 6.98 \sim 6.84(\mathrm{~m}, 2 \mathrm{H}), 4.74$ (s, 2H), 4.64 (s, 2H), 3.84 (s, 3H) ${ }^{13} \mathrm{C}$ NMR (101 MHz, $\left.\mathrm{CDCl}_{3}\right) \delta: 183.7,167.3,159.8,151.5,149.1,138.1,130.2$, 129.4, 126.1, 124.6, 114.6, 102.1, 55.4, 55.3, 51.3; HRMS (ESI) calcd for $\mathrm{C}_{17} \mathrm{H}_{15} \mathrm{Cl}_{2} \mathrm{~N}_{2} \mathrm{O}_{2} \mathrm{~S}_{2}[\mathrm{M}+\mathrm{H}]^{+}$412.9952, found 412.9938 .

4-氯-5-(((6-氯吡啶-3-基)甲基)(4-氟芐基)氨基)3H-1,2-二硫醇-3-酮(3i): 橘黄色油状液体, 产率 71\%. ${ }^{1} \mathrm{H}$ NMR (400 MHz, $\left.\mathrm{CDCl}_{3}\right) \delta: 8.24(\mathrm{~d}, J=2.5 \mathrm{~Hz}, 1 \mathrm{H}), 7.59$ (dd, $J=8.2,2.6 \mathrm{~Hz}, 1 \mathrm{H}), 7.36(\mathrm{~d}, J=8.2 \mathrm{~Hz}, 1 \mathrm{H}), 7.26 \sim$ $7.21(\mathrm{~m}, 2 \mathrm{H}), 7.14 \sim 7.04(\mathrm{~m}, 2 \mathrm{H}), 4.72(\mathrm{~s}, 2 \mathrm{H}), 4.67(\mathrm{~s}$, $2 \mathrm{H}) ;{ }^{13} \mathrm{C}$ NMR $\left(101 \mathrm{MHz}, \mathrm{CDCl}_{3}\right) \delta: 183.7,167.2,162.7$ (d, $J=248.3 \mathrm{~Hz}), 151.7,149.1,138.1,130.2,130.0,129.7$,
129.6, 124.7, 116.4, 116.1, 102.8, 55.1, 51.8; HRMS (ESI) calcd for $\mathrm{C}_{16} \mathrm{H}_{12} \mathrm{Cl}_{2} \mathrm{FN}_{2} \mathrm{OS}_{2}[\mathrm{M}+\mathrm{H}]^{+}$400.9752, found 400.9743.

4-氯-5-(((6-氯吡啶-3-基)甲基)(4-甲基苄基)氨基)$3 H$-1,2-二硫醇-3-酮 $(\mathbf{3} \mathbf{j})$ : 棕黄色油状液体，产率 $56 \%$. ${ }^{1} \mathrm{H}$ NMR $\left(400 \mathrm{MHz}, \mathrm{CDCl}_{3}\right) \delta: 8.25(\mathrm{~d}, J=2.5 \mathrm{~Hz}, 1 \mathrm{H})$, 7.60 (dd, $J=8.3,2.6 \mathrm{~Hz}, 1 \mathrm{H}), 7.37(\mathrm{~d}, J=8.2 \mathrm{~Hz}, 1 \mathrm{H})$, $7.22(\mathrm{~d}, J=8.0 \mathrm{~Hz}, 2 \mathrm{H}), 7.15(\mathrm{~d}, J=8.0 \mathrm{~Hz}, 2 \mathrm{H}), 4.75(\mathrm{~s}$, $2 \mathrm{H}), 4.67(\mathrm{~s}, 2 \mathrm{H}), 2.39$ (d, $J=3.5 \mathrm{~Hz}, 3 \mathrm{H}) ;{ }^{13} \mathrm{C}$ NMR $(101$ $\left.\mathrm{MHz}, \mathrm{CDCl}_{3}\right) \delta: 183.7,167.3,151.5,149.1,138.6,138.2$, 131.2, 130.2, 129.9, 127.9, 124.6, 102.0, 55.7, 51.5, 21.2; HRMS (ESI) calcd for $\mathrm{C}_{17} \mathrm{H}_{15} \mathrm{Cl}_{2} \mathrm{~N}_{2} \mathrm{OS}_{2}[\mathrm{M}+\mathrm{H}]$ 397.0003, found 396.9994.

5-(芐基(((6-氯吡啶基-3-基)甲基)氨基)-4-氯-3H-1,2二硫醇-3-酮(3k): 深黄色油状液体, 产率 49\%. ${ }^{1} \mathrm{H}$ NMR $\left(400 \mathrm{MHz}, \mathrm{CDCl}_{3}\right) \delta: 8.26(\mathrm{~d}, J=2.5 \mathrm{~Hz}, 1 \mathrm{H}), 7.61(\mathrm{dd}$, $J=8.3,2.6 \mathrm{~Hz}, 1 \mathrm{H}), 7.42(\mathrm{~d}, J=1.7 \mathrm{~Hz}, 1 \mathrm{H}), 7.41 \sim 7.36$ (m, 3H), 7.30 7.24 (m, 2H), 4.77 (s, 2H), 4.72 (s, 2H); ${ }^{13} \mathrm{C}$ NMR (101 MHz, $\mathrm{CDCl}_{3}$ ) $\delta: 183.7,167.3,151.6,149.1$, 138.1, 134.4, 130.1, 129.2, 128.7, 127.9, 124.7, 102.3, 55.8, 51.7; HRMS (ESI) calcd for $\mathrm{C}_{16} \mathrm{H}_{13} \mathrm{Cl}_{2} \mathrm{~N}_{2} \mathrm{OS}_{2}[\mathrm{M}+\mathrm{H}]^{+}$ 382.9846 , found 382.9838 .

4-氯-5-((2-氯苄基)((6-氯吡啶-3-基)甲基)氨基)-3H1,2-二硫醇-3-酮(3I): 棕黄色油状液体, 产率 $63 \% .{ }^{1} \mathrm{H}$ NMR (400 MHz, $\left.\mathrm{CDCl}_{3}\right) \delta: 8.29$ (d, $\left.J=2.5 \mathrm{~Hz}, 1 \mathrm{H}\right), 7.63$ (dd, $J=8.3,2.5 \mathrm{~Hz}, 1 \mathrm{H}), 7.44 \sim 7.41(\mathrm{~m}, 1 \mathrm{H}), 7.37$ (d, $J=$ $8.2 \mathrm{~Hz}, 1 \mathrm{H}$ ), 7.31 (ddt, $J=9.7,5.7,3.4 \mathrm{~Hz}, 3 \mathrm{H}$ ), 4.83 (s, $2 \mathrm{H}), 4.81(\mathrm{~s}, 2 \mathrm{H}) ;{ }^{13} \mathrm{C}$ NMR $\left(101 \mathrm{MHz}, \mathrm{CDCl}_{3}\right) \delta: 183.7$, $167.1,151.6,148.9,138.0,133.6,132.1,130.2,130.1$, $129.9,129.1,127.4,124.7,102.4,53.5,52.5$; HRMS (ESI) calcd for $\mathrm{C}_{16} \mathrm{H}_{12} \mathrm{Cl}_{3} \mathrm{~N}_{2} \mathrm{OS}_{2}[\mathrm{M}+\mathrm{H}]^{+}$416.9457, found 416.9452

4-氯-5-(((6-氯吡啶-3-基)甲基)(2-氟乙基)氨基)-3H1,2-二硫醇-3-酮(3m)：暗红色油状液体，产率 $54 \% .{ }^{1} \mathrm{H}$ NMR (400 MHz, $\left.\mathrm{CDCl}_{3}\right) \delta: 8.36(\mathrm{~d}, J=2.5 \mathrm{~Hz}, 1 \mathrm{H}), 7.67$ (dd, $J=8.3,2.6 \mathrm{~Hz}, 1 \mathrm{H}), 7.38$ (d, $J=8.2 \mathrm{~Hz}, 1 \mathrm{H}), 4.90$ (s, $2 \mathrm{H}), 4.72$ (dt, $J=47.2,4.5 \mathrm{~Hz}, 2 \mathrm{H}), 3.95(\mathrm{dt}, J=26.5,4.5$ $\mathrm{Hz}, 2 \mathrm{H}) ;{ }^{13} \mathrm{C}$ NMR $\left(101 \mathrm{MHz}, \mathrm{CDCl}_{3}\right) \delta: 183.53,166.39$, $151.62,148.96,138.03,130.12,124.71,102.40,82.38$ (d, $J=171.7 \mathrm{~Hz}), 54.22,52.57$ (d, $J=19.9 \mathrm{~Hz})$; HRMS (ESI) calcd for $\mathrm{C}_{11} \mathrm{H}_{10} \mathrm{Cl}_{2} \mathrm{FN}_{2} \mathrm{OS}_{2}[\mathrm{M}+\mathrm{H}]^{+}$338.9596, found 338.9589 .

4-氯-5-(((6-氯吡啶-3-基)甲基)(2-氯乙基)氨基)3H-1,2-二硫醇-3-酮(3n): 浅黄绿色固体, 产率 60\%. m.p. $85 \sim 87{ }^{\circ} \mathrm{C} ;{ }^{1} \mathrm{H}$ NMR $\left(400 \mathrm{MHz}, \mathrm{CDCl}_{3}\right) \delta: 8.37$ (d, $J=2.5$ 
$\mathrm{Hz}, 1 \mathrm{H}), 7.66$ (dd, $J=8.3,2.5 \mathrm{~Hz}, 1 \mathrm{H}), 7.40(\mathrm{~d}, J=8.2 \mathrm{~Hz}$, $1 \mathrm{H}), 4.92(\mathrm{~s}, 2 \mathrm{H}), 3.97$ (t, $J=6.2 \mathrm{~Hz}, 2 \mathrm{H}), 3.74$ (t, $J=6.2$ $\mathrm{Hz}, 2 \mathrm{H}) ;{ }^{13} \mathrm{C}$ NMR $\left(101 \mathrm{MHz}, \mathrm{CDCl}_{3}\right) \delta: 183.55,165.97$, $151.77,148.84,137.93,130.05,124.80,102.78,54.44$, 53.79, 41.34; HRMS (ESI) calcd for $\mathrm{C}_{12} \mathrm{H}_{9} \mathrm{Cl}_{3}-\mathrm{N}_{2} \mathrm{OS}_{2}$ [M $+\mathrm{H}]^{+} 354.9300$, found 354.9300 .

\subsection{3 目标化合物 $\mathbf{4 a} \sim \mathbf{4 e}$ 的合成}

$50 \mathrm{~mL}$ 圆底烧瓶中依次加入中间体 $2(4 \mathrm{mmol})$ 、二 氯-1,2-二硫环戊烯酮 $(2.7 \mathrm{mmol})$ 与 $3 \mathrm{~mL} \mathrm{DMF}$, 搅拌使其 完全溶解. 混合液加入无水碳酸钾 $0.35 \mathrm{~g}$, 室温摚拌, TLC 跟踪反应. 反应结束后向反应液中加入 $50 \mathrm{~mL}$ 冰 水, 抽滤, 将滤饼溶于 $50 \mathrm{~mL}$ 二氯甲烷, 经 $1 \mathrm{~mol} / \mathrm{L}$ 盐酸 溶液、饱和碳酸氢钠溶液和清水洗涤, 干燥后减压浓缩, 粗产品经柱层析分离 $[V$ (二氯甲烷 $): V($ 乙酸乙酯 $)=$ 10：1]提纯得到目标化合物 $\mathbf{4 a \sim 4 e}$.

4-氯-5-(((2-氯噻唑-5-基)甲基)(甲基)氨基)-3H-1,2二硫醇-3-酮 (4a): 淡黄色固体, 产率 70\%. m.p. 82 $84{ }^{\circ} \mathrm{C} ;{ }^{1} \mathrm{H}$ NMR $\left(400 \mathrm{MHz}, \mathrm{CDCl}_{3}\right) \delta: 7.51(\mathrm{~d}, J=0.9 \mathrm{~Hz}$, 1H), 4.94 (d, $J=0.9 \mathrm{~Hz}, 2 \mathrm{H}), 3.17$ (s, 3H); ${ }^{13} \mathrm{C}$ NMR $(101$ $\left.\mathrm{MHz}, \mathrm{CDCl}_{3}\right) \delta: 183.3,167.0,153.5,141.0,134.7,100.8$, 50.5, 39.0; HRMS (ESI) calcd for $\mathrm{C}_{8} \mathrm{H}_{7} \mathrm{Cl}_{2} \mathrm{~N}_{2} \mathrm{OS}_{3}[\mathrm{M}+\mathrm{H}]^{+}$ 312.9098, found 312.9088 .

4-氯-5-(((2-氯噻唑-5-基)甲基)(乙基)氨基)-3H-1,2二硫醇-3-酩(4b): 暗黄色固体, 产率 53\%. m.p. 73 $75{ }^{\circ} \mathrm{C} ;{ }^{1} \mathrm{H}$ NMR $\left(400 \mathrm{MHz}, \mathrm{CDCl}_{3} \delta: 7.50(\mathrm{~d}, J=1.0 \mathrm{~Hz}\right.$, $1 \mathrm{H}), 4.93(\mathrm{~d}, J=1.0 \mathrm{~Hz}, 2 \mathrm{H}), 3.60$ (q, $J=7.1 \mathrm{~Hz}, 2 \mathrm{H}), 1.32$ (t, $J=7.1 \mathrm{~Hz}, 3 \mathrm{H}) ;{ }^{13} \mathrm{C}$ NMR $\left(101 \mathrm{MHz}, \mathrm{CDCl}_{3}\right) \delta: 183.5$, 165.9, 153.3, 140.7, 135.4, 101.2, 48.0, 47.0, 13.5; HRMS (ESI) calcd for $\mathrm{C}_{9} \mathrm{H}_{9} \mathrm{Cl}_{2} \mathrm{~N}_{2} \mathrm{OS}_{3}[\mathrm{M}+\mathrm{H}]^{+} 326.9254$, found 326.9245 .

5-(苄基(((2-2-氯噻唑-5-基)甲基)氨基)-4-氯-3H-1,2二硫基-3-酮(4c)：淡黄色油状液体，产率 50\%. m.p. 98 $100{ }^{\circ} \mathrm{C} ;{ }^{1} \mathrm{H}$ NMR $\left(400 \mathrm{MHz}, \mathrm{CDCl}_{3}\right) \delta: 7.45(\mathrm{~d}, J=6.5 \mathrm{~Hz}$, $1 \mathrm{H}), 7.43 \sim 7.37(\mathrm{~m}, 3 \mathrm{H}), 7.34 \sim 7.29(\mathrm{~m}, 2 \mathrm{H}), 4.87(\mathrm{~s}$, 2H), $4.71(\mathrm{~s}, 2 \mathrm{H}) ;{ }^{13} \mathrm{C}$ NMR (101 MHz, $\left.\mathrm{CDCl}_{3}\right) \delta: 183.6$, $166.8,153.3,141.2,134.6,134.2,129.3,128.7,127.9$, 102.9, 55.1, 47.2; HRMS (ESI) calcd for $\mathrm{C}_{14} \mathrm{H}_{11} \mathrm{Cl}_{2} \mathrm{~N}_{2} \mathrm{OS}_{3}$ $[\mathrm{M}+\mathrm{H}]^{+}$388.9411, found 388.9403 .

4-氯-5-(((2-氯噻唑-5-基)甲基)(4-甲基芐基)氨基)3H-1,2-二硫醇-3-酮(4d): 浅棕色固体, 产率 50\%. m.p. $111 \sim 113{ }^{\circ} \mathrm{C} ;{ }^{1} \mathrm{H}$ NMR $\left(400 \mathrm{MHz}, \mathrm{CDCl}_{3}\right) \delta: 7.36(\mathrm{~s}, 1 \mathrm{H})$, 7.22 (d, $J=7.9 \mathrm{~Hz}, 2 \mathrm{H}), 7.18$ (d, $J=7.9 \mathrm{~Hz}, 2 \mathrm{H}), 4.82$ (s, 2H), $4.64(\mathrm{~s}, 2 \mathrm{H}), 2.38(\mathrm{~s}, 3 \mathrm{H}) ;{ }^{13} \mathrm{C}$ NMR (101 MHz, $\left.\mathrm{CDCl}_{3}\right) \delta: 183.7,166.87,153.3,141.2,138.7,134.6,131.1$, 129.9, 128.0, 102.8, 54.9, 47.0, 21.2; HRMS (ESI) calcd for $\mathrm{C}_{15} \mathrm{H}_{13} \mathrm{Cl}_{2} \mathrm{~N}_{2} \mathrm{OS}_{3}[\mathrm{M}+\mathrm{H}]^{+}$402.9567, found 402.9556 .

4-氯-5-(((2-氯噻唑-5-基)甲基)(4-氟芐基)氨基)- $3 \mathrm{H}$ 1,2-二硫醇-3-酮(4e): 浅红色固体, 产率 54\%. m.p. 99 $101{ }^{\circ} \mathrm{C} ;{ }^{1} \mathrm{H}$ NMR $\left(400 \mathrm{MHz}, \mathrm{CDCl}_{3}\right) \delta: 7.38(\mathrm{~s}, 1 \mathrm{H})$, $7.31 \sim 7.26(\mathrm{~m}, 3 \mathrm{H}), 7.13 \sim 7.08(\mathrm{~m}, 2 \mathrm{H}), 4.82(\mathrm{~d}, J=1.2$ $\mathrm{Hz}, 2 \mathrm{H}), 4.66(\mathrm{~s}, 2 \mathrm{H}) ;{ }^{13} \mathrm{C}$ NMR $\left(101 \mathrm{MHz}, \mathrm{CDCl}_{3}\right) \delta$ : 183.7, 166.8, 162.7 (d, $J=248.3 \mathrm{~Hz}), 153.4,141.2,134.5$, 130.1, 129.7, 129.7, 116.3, 116.2, 103.5, 54.4, 47.4; HRMS (ESI) calcd for $\mathrm{C}_{14} \mathrm{H}_{10} \mathrm{Cl}_{2} \mathrm{FN}_{2} \mathrm{OS}_{3}[\mathrm{M}+\mathrm{H}]^{+}$406.9316, found 406.9310 .

\subsection{4 化合物的生物活性测试}

选用苜宿蚜虫和稻飞風为供试害虫, 氟吡呋喃酮 (Flupyradifurone)为对照药剂, 含 DMF 的吐温-80 水溶液 作为空白对照. 将对照药剂和待测化合物用 DMF 溶解, 再加入含吐温-80 $(1 \mathrm{~g} / \mathrm{L})$ 的水溶液稀释至所需浓度. 每 个处理 3 次重复, 并设置空白对照. 用药液浸渍法, 将事 先挑选的供试害虫在药液中浸渍 $5 \mathrm{~s}$ 取出, 晾干后置于 $(25 \pm 1){ }^{\circ} \mathrm{C}$ 下湿润的培养血中. $48 \mathrm{~h}$ 后检查结果, 轻触虫 体, 无反应或者反应迟缓不能正常爬行者均判定为死 $亡^{[17]}$.

\section{校正死亡率 $(\%)=($ 样品死亡率一空白对照死亡率 $) /$}

(1一空白对照死亡率) $\times 100 \%$

空白对照死亡率小于 $5 \%$ 可不必校正, 大于 $20 \%$ 为 无效试验.

辅助材料(Supporting Information) 化合物 $3 \mathbf{a} \sim 3 \mathbf{n}$, 4a $\sim 4 \mathrm{e}$ 的 ${ }^{1} \mathrm{H} N M R,{ }^{13} \mathrm{C}$ NMR 和 HRMS 图谱. 这些材料 可以免费从本刊网站(http://sioc-journal.cn/)上下载.

\section{References}

[1] Talley, T. T.; Harel, M.; Hibbs, R. E.; Radi, C. Z.; Tomizawa, M.; Casida, J. E.; Taylor, P. Proc. Natl. Acad. Sci. U. S. A. 2008, 105, 7606.

[2] Chen, L.; Wang, B. Q.; Zhao, Y. C.; Yan, S. J.; Lin, J. Chin. J. Org. Chem. 2017, 37, 1433 (in Chinese).

(陈亮, 王保取, 赵宇澄, 严胜娇, 林军, 有机化学, 2017, 37, 1433.)

[3] Jeschke, P.; Nauen, R. Pest Manage. Sci. 2008, 64, 1084.

[4] Yu, L. World Pestic. 2018, 24, 45 (in Chinese). (余露, 世界农药, 2018, 24, 45).

[5] Bartlett, A. J.; Hedges, A. M.; Intini, K. D.; Brown, L. R.; Maisonneuve, F. J.; Robinson, S. A.; Gillis, P. L.; Solla, S. R. Environ. Pollut. 2018, 238, 63.

[6] Nauen, R.; Jeschke, P.; Velten, R.; Beck, M. E.; EbbinghausKintscher, U.; Thielert, W.; Wölfel, K.; Haas, M.; Kunz, K.; Raupach, G. Pest Manage. Sci. 2015, 71, 8507.

[7] Jeschke, P.; Nauen, R.; Gutbrod, O.; Beck, M. E.; Matthiesen, S.; Haas, M.; Velten, R. Pestic. Biochem. Physiol. 2015, 121, 31.

[8] Han, L. World Pestic. 2016, 38, 9 (in Chinese). (韩醴, 世界农药, 2016, 38, 9).

[9] Zhang, Y. X. World Pestic. 2015, 37, 62 (in Chinese). 
(张翼鲾，世界农药, 2015, 37, 62).

[10] Zhu, Y. J.; Gu, L. L. Mod. Agrochem. 2018, 17, 21 (in Chinese). (朱亚娟, 顾林玲, 现代农药, 2018, 17, 21.)

[11] Chen, X. D.; Seo, M.; Stelinski, L. L. Crop Prot. 2017, 98, 102.

[12] Tian, P. Y.; Liu, D. Y.; Liu, Z. J.; Shi, J.; He, W. J.; Qi, P. Y.; Chen, J. X.; Song, B. A. Pest Manage. Sci. 2018, 75, 427.

[13] Wang, X. H.; Li, J. G.; Wang, G. J.; Han, X. Z.; Li, D. M.; Tian, Z. Z. Chem. Res. Chin. Univ. 2019, 35, 799 .

[14] He, X., Reeve, A. M.; Desai, U. R.; Kellogg, G. E.; Reynolds, K. A.
Antimicrob. Agents Chemother. 2004, 48, 3093.

[15] Fracaroli, A. M.; Kreiker, J.; Rossi, R. H.; Granados, A. M. ARKIVOC 2007, iv, 279.

[16] Sulur, G.; Manjunatha, K.; Reddy, V.; Rajappa, S. Tetrahedron Lett. 1990, 31, 1327.

[17] Chen, Y. B.; Fan, J.; Xia, S.; Cheng, J. G.; Xu, X. Y.; Li, Z. Chin. J. Org. Chem. 2014, 34, 409 (in Chinese).

(陈寅波，范杰，夏爽，程家高，徐晓勇，李忠，有机化学，2014， 34, 409.)

(Lu, Y.) 